1 Hacettepe Journal of Mathematics and Statistics

$\bigcap$ Volume 47 (5) (2018), 1071-1093

\title{
Distance property of chemical graphs
}

\author{
Sakander Hayat*†, Shahzad Ahmad ${ }^{\ddagger}$, Hafiz Muhammad Umair ${ }^{\S}$ and Shaohui \\ Wang 9
}

\begin{abstract}
We have developed a rigorous computational technique to compute exact analytic expressions for a number of distance-based topological indices of chemical graphs. There are two main advantages of our technique over existing techniques of similar nature: first, our technique is significantly diverse as it also covers the Wiener index and eccentricitybased topological indices besides Szeged-like indices, and secondly we have considerably reduced the algorithmic and computational complexity in comparison to previous techniques. Our proposed technique generates certain vertex and edge partitions of a graph which are essential in computing the exact analytical formulas of distance-based and eccentricity-based indices. To ensure the applicability of our technique, we have computed various distance-based and eccentricity-based topological indices for certain infinite families of polyomino chain system. Moreover, we find analytical exact expressions of certain degree-based topological indices for these polyomino chains. These topological indices can be obtained as a by-product of our technique.
\end{abstract}

Keywords: Combinatorial algorithms, Distance-based topological indices, Eccentricity-based topological indices, Degree-based topological indices, Polyomino chain system.

Mathematics Subject Classification (2010): 05C12, 05C90

Received : 28.02.2017 Accepted : 14.06.2017 Doi : 10.15672/HJMS.2017.487

*School of Mathematical Sciences, University of Science and Technology of China (USTC), Hefei, 230026, China., Email: sakander@mail.ustc.edu.cn

$\dagger$ Corresponding Author.

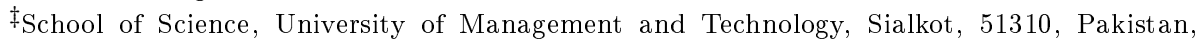
Email: shahzad.ahmed@skt.umt.edu.pk

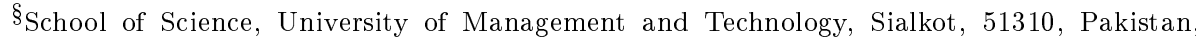
Email: jawad.math@gmail.com

IDepartment of Mathematics and Computer Science, Adelphi University, Garden City, NY 11530, USA, Email: shaohuiwang@yahoo.com 


\section{Introduction}

Graph theory, especially distance-based metrics has been applied to several wideranging physico-chemical applications particularly in the characterization and modeling of chemical structures. Graph-theoretical applications to chemistry and drug research originate from the fact that the properties and activities of molecules are intimately related to their underlying connectivities. As graph theory is concerned with topological connectivity of a chemical structure and its characterization, a number of research papers have been devoted for exploring the relationships between chemical properties and graph-theoretical based topological indices over the years, see [4] and references therein. Cheminformatics is an emerging field of research which integrates the concepts from chemistry, mathematics and information science. The research in this field is concerned with the quantitative structure-activity (QSAR) and structure-property (QSPR) relationships, as the properties of molecules are derivable from their chemical structures. The most important step in QSAR/QSPR is to quantify the molecular structures so as to build a correlation model between the chemical structures of various chemical compounds and the corresponding chemical and biological properties [7, 22].

Arockiaraj et al. [4] proposed a rigorous mathematical and computational technique to compute exact analytical expressions of a number of distance-degree related topological indices of partial cubes. The partial cubes are a reasonably big class of graphs which are actually isometrically embeddable on $\ell_{1}$-space or more precisely, on hypercubes. Note that the partial cubes have a non-empty intersection with the class of chemical graphs. For example, the polyomino chains which are considered in this paper, belong to the intersection of these two classes of graphs. Ashrafi et al. [2] developed a computational technique to compute Szeged, PI and revised Szeged indices of chemical graphs and computed these indices for an infinite family of fullerenes. In this paper, we generalize the work by Arockiaraj et al. and Ashrafi et al. in the sense that our computational technique is valid for chemical graphs and covers various distance-based, eccentricity-based and degree-based topological indices. We reduce the computational complexity significantly as well of the work by Ashrafi et al. The motivation to consider the polyomino chains comes from Imran et al. [6] where they studied the counting related polynomials and indices of these chains.

Polyominoes are the combinatorial and geometric shapes made by connecting certain numbers of squares (4-cycles), each joined together with at least one other square along an edge. In enumerative combinatorics, they might call this connected a "rookwise connection". Polyomino patterns are actually examples of combinatorial geometry, that branch of mathematics dealing with the ways in which geometrical shapes can be combined. It is a frequently neglected aspect of mathematics because it seems to have few general methods, and because in it systematic rules have not replaced ingenuity as the key to discovery. Many of the design problems in practical engineering are combinatorial in nature, especially when standard components or shapes are to be fitted together in some optimal fashion [13].

A $k$-polyomino system is a finite 2-connected plane graph such that each interior face (also called cell) is surrounded by a regular $4 k$-cycle of length one. In other words, it is an edge-connected union of cells [24]. This polyomino system divides the plane into one infinite external region and a number of finite internal, all internal region must be squares. Polyominoes have a long and rich history, we convey for the origin polyominoes. A polyomino chain is a polyomino system, in which the joining of the centers of its adjacent regular forms a path $c_{1}, c_{2}, \ldots, c_{n}$, where $c_{i}$ is the center of the $i$-th square. A square of a polyomino chain has either one or two neighboring squares. If a square has one neighboring square, it is called terminal, if it has two neighboring squares having no 


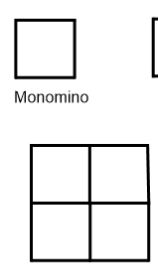

Square Tetromin

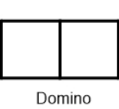

Domino

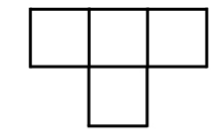

T Tetromino

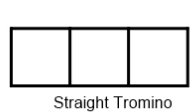

traight Tromino

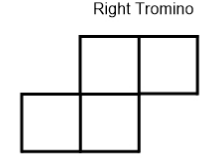

Skew Tetromino
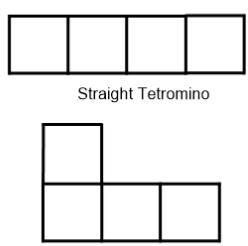

L Tetromino

Figure 1. The simpler polyominoes.

vertex of degree 2, it is called medial, and if it has two neighboring squares such that it has a vertex of degree 2, it is called kink. Every polyomino chain of dimension $n$ has a unit such that it contains $n$ number of units [43]. We denote the polyomino chain system by $P_{n}^{k}$, and $k=k^{\prime}+m+t$, where $k^{\prime}$ is the number of kinks, $m$ is the number of medials and $t$ is the number of terminals in a unit of polyomino chain. Figure 3 depicts the different polyomino chains with their units as dotted part. In Figure 2, a general representation of polyomino chain system $P_{n}^{k}$ is depicted.

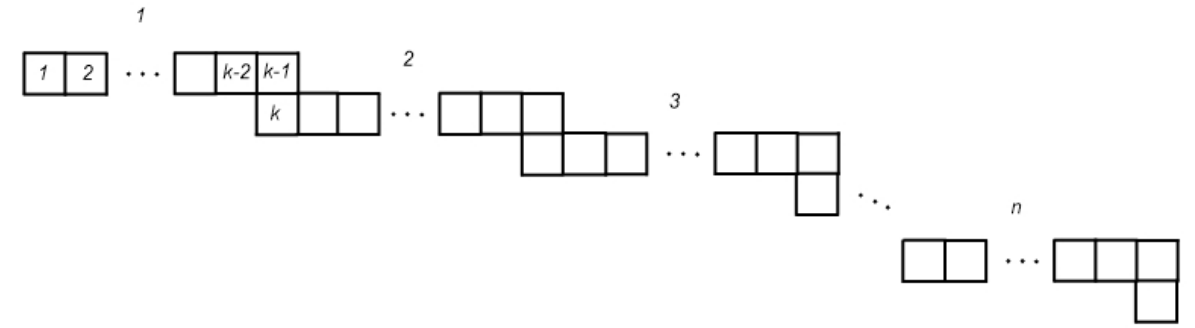

Figure 2. A general representation of polyomino chain system $P_{n}^{k}$.

In chemical graph theory, a topological index/descritptor is a numerical quantity which is evaluated globally based on the chemical structure of underlying chemical compound. The corresponding chemical structures are reported in the literature to be named as the chemical/molecular graphs of underlying compounds. Thus topological indices provide for quantitative characterization of a graph that is topologically invariant to labeling and at the same time differentiating properties of isomers. There are a wide range of topological indices which have been defined and investigated so far. Among them, distance-based and degree-based indices play a vital in chemical sciences and computing these topological indices is one of the recent areas of research in chemical graph theory and reticular chemistry.

\section{Preliminaries}

In this paper, by graph/network we mean a connected, simple and finite graph. For a graph $G$, we denote by $V(G)$ and $E(G)$ the set of vertices and set of edges respectively. A graph is said to be a chemical graph, if its vertices represent the atoms and edges represent the bonds between them. Note that a vertex can not have degree more than four in a chemical graph. A vertex is called a cut vertex, if its deletion makes the graph disconnected. A bi-connected graph is a connected graph which has no cut vertex. In a 


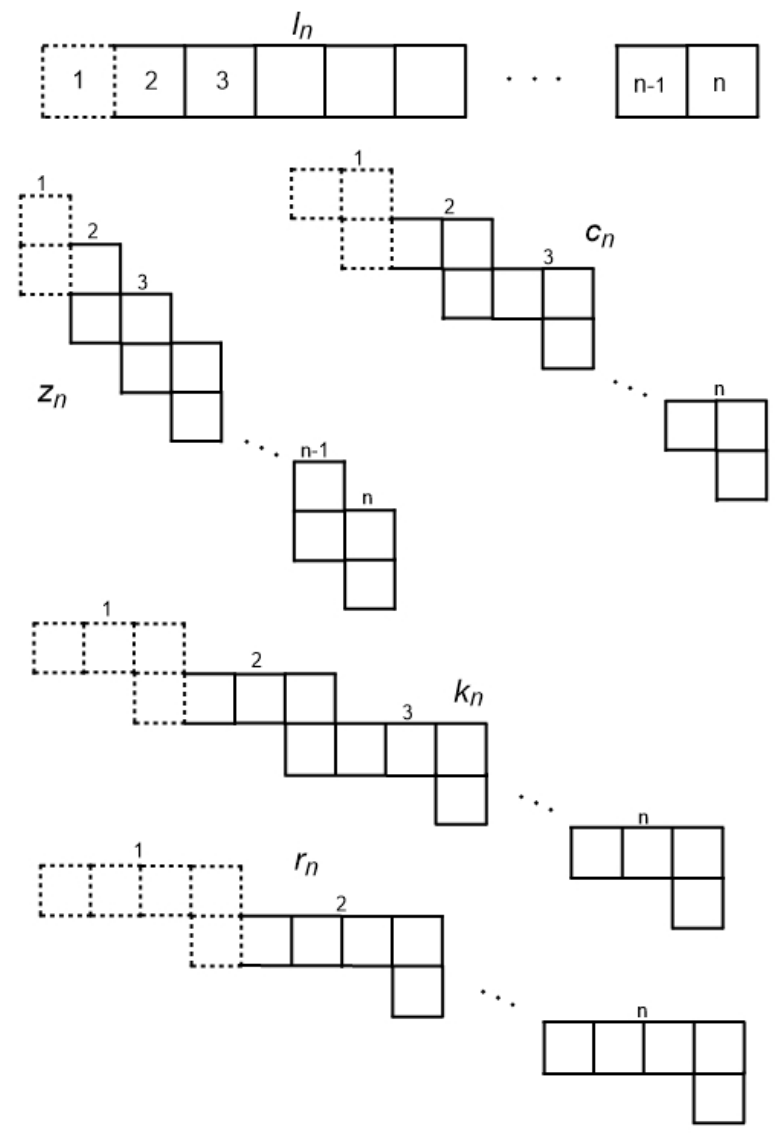

Figure 3. Different polyomino chains with dotted parts as their units.

bi-connected graph, if we remove any vertex, the resulting graph will remain connected. A cubic graph is a graph in which every vertex has degree three.

For a graph $G$, a function $\sigma$ from $G$ to itself is an automorphism if and only if $\sigma$ is one-to-one and both $\sigma$ and its inverse are adjacency preservers in $G$. If a property $p$ is preserved under every automorphism, its is called a topological property. A topological index is a numerical quantity which describes a topological property and should have significant application in chemistry. The topological distance between two vertices $u$ and $v$ in a chemical graph $G$ is the length of the shortest distance between $u$ and $v$. A distance-based topological index is a topological index which is defined by some distance function $d_{G}$.

In 1947, an American chemist Harold Wiener [41] discovered a first topological index while investigating the boiling points of Alkanes. He named this topological index path number which later on became the Wiener index. For a graph $G$, the Wiener index $W(G)$ is defined as

$$
W(G)=\sum_{\{u, v\} \subset E(G)} d(u, v) .
$$


The transmission $\operatorname{Tr}(v)$ of a vertex $v$ in $G$ is the sum of the distances from $v$ to all other vertices in $G$, i.e.,

$$
\operatorname{Tr}(v)=\sum_{u \in V} d(u, v) \text {. }
$$

Note that $W(G)=\frac{1}{2} \sum_{1 \leq i \leq n} \operatorname{Tr}\left(v_{i}\right)$. After Wiener, many topological indices where proposed by chemist and also by mathematicians.

The first degree based topological index is the Randić index [34] introduced by Milan Randić in 1975. The Randić index is defined as

$$
\chi(G)=\sum_{u v \in E(G)} \frac{1}{\sqrt{d_{u} d_{v}}} .
$$

The Randić index has been closely correlated with many chemical properties and found to parallel the boiling point and Kovats constants.

The widely used connectivity topological index is the Atom-Bond Connectivity $(A B C)$ index introduced by Estrada et. al [11] and is defined as

$$
A B C(G)=\sum_{u v \in E(G)} \sqrt{\frac{d_{u}+d_{v}-2}{d_{u} d_{v}}} .
$$

The ABC index provides a good model for the stability of linear and branched alkanes as well as the strain energy of cycloalkanes.

The fourth version of $A B C$ index $\left(A B C_{4}\right)$ is introduced by Ghorbani et. al [15] and is defined as

$$
A B C_{4}(G)=\sum_{u v \in E(G)} \sqrt{\frac{S_{u}+S_{v}-2}{S_{u} S_{v}}} .
$$

The Geometric-Arithmetic $(G A)$ index which is introduced by Vukičević et. al [38] and defined as

$$
G A(G)=\sum_{u v \in E(G)} \frac{2 \sqrt{d_{u} d_{v}}}{\left(d_{u}+d_{v}\right)} .
$$

For certain physico-chemical properties, the predictive power of GA index is somewhat better than predictive power of the Randić connectivity index.

Recently fifth version of $G A$ index $\left(G A_{5}\right)$ is proposed by Graovac et. al [14] and defined as

$$
G A_{5}(G)=\sum_{u v \in E(G)} \frac{2 \sqrt{S_{u} S_{v}}}{\left(S_{u}+S_{v}\right)} .
$$

For a graph $G$, the space $\left(V(G), d_{G}\right)$ is a metric space and therefore the topological indices defined based on distance function $d_{G}$ reveal significant topological/structural information about the under considered chemical compound. Padmakar-Khadiakar [23] introduced the Padmakar-Ivan (PI) index. To better understand the definition of the PI index, we first define the quantities $\nu(e \mid u), \nu(e \mid v)$ and $\nu(e \mid 0)$ for an edge $e=u v$ of a graph $G$. They are defined as follows:

$$
\begin{aligned}
& \nu(e \mid u)=|\{x \in V(G) \mid d(x, u)<d(x, v)\}| \\
& \nu(e \mid v)=|\{x \in V(G) \mid d(x, v)<d(x, u)\}| \\
& \nu(e \mid 0)=|\{x \in V(G) \mid d(x, v)=d(x, u)\}| .
\end{aligned}
$$

We denote the triplet $(\nu(e \mid u), \nu(e \mid v), \nu(e \mid 0))$ by $\mathcal{N}(e)$, for an edge $e=u v \in E(G)$. 
The PI index is defined as $P I(G)=\sum_{e=u v \in E(G)}[\nu(e \mid u)+\nu(e \mid v)]$. The Wiener index was first defined only for trees as Harold Wiener was working with alkanes and the chemical graphs corresponding to them are trees. For a tree $T$, the Wiener index $W(T)$ can be defined as $W(T)=\sum_{e=u v \in E(G)} \nu(e \mid u) \nu(e \mid u)$. To extend this concept for general graphs, Ivan Gutman [17] defined a similar topological index for a general graph with significantly better correlation for non-cyclic chemical compounds. The Szeged index of a graph $G$, is defined as $S z(G)=\sum_{e=u v \in E(G)} \nu(e \mid u) \nu(e \mid v)$. For more mathematical information, we consult the reader to paper [18].

There is a modification of Szeged index to find better correlation in chemistry, discovered by Milan Randić [35]. Later, this modification was named the revised Szeged index. It is defined as $R S z(G)=\sum_{e=u v \in E(G)}\left[\nu(e \mid u)+\frac{\nu(e \mid 0)}{2}\right]\left[\nu(e \mid v)+\frac{\nu(e \mid 0)}{2}\right]$. For mathematical as well as chemical meaning and significance of these topological indices, we refer the reader to papers [3, 33, 42]. For application of the Szeged and the revised Szeged indices for carbon nanotubes, see for example, [10].

Now we define the eccentricity-based topological indices of a graph $G$. The eccentricity of a vertex $v$ denoted by $\varepsilon(u)$, is the maximum distance between $v$ and any other vertex of $G$. The largest eccentricity of a vertex in a graph is called its diameter $d(G)$, whereas the minimum eccentricity among all is called the radius $r(G)$ of a graph. The eccentricconnectivity index [36] $\xi(G)$ of $G$ is defined as $\xi(G)=\sum_{u \in V(G)} d_{u} \varepsilon(u)$. When the valencies of a vertex is not considered, the total eccentricity index $\zeta(G)=\sum_{u \in V(G)} \varepsilon(u)$. We encourage the reader to consult papers [9] and [44] for the chemical meaning and the mathematical properties of these topological indices. We also refer the papers $[1,19,21]$ and references therein. For mathematical and extremal properties of other related topological indices for different families of graphs, we refer the work by Liu et al. [25, 26, 27, 28, 29].

\section{Proposed computational technique}

In this section, we present our rigorous mathematical and computational techniques to obtain exact analytical expressions of a number of distance-based topological indices. Our proposed techniques are based on certain mathematical and chemical computer softwares like Hyperchem [20], Topocluj [8] and MATLAB [30]. There are some computational techniques developed for chemical graphs in the literature proposed by Mehranian [31] and Mottaghi et al. [32] which uses GAP [12] to compute Szeged, PI and revised Szeged indices for chemical graphs. In this paper, we extend the previous techniques by enhancing its applicability to various eccentricity-based topological indices such as eccentric connectivity index, total eccentricity index and eccentricity-based Zagreb indices. Moreover, we reduce the computational complexity of the previous techniques by introducing the MATLAB instead of GAP whose user interface is not much user-friendly.

Before we proceed further to present the step-by-step procedure of our method, we give some necessary definitions which are subsequently used in the later sections. The adjacency matrix of graph $G$, denoted by $A_{G}$, is the $n \times n$ symmetric matrix defined as

$$
A_{G}(i, j)= \begin{cases}1, & i j \in E(G) \\ 0, & \text { Otherwise }\end{cases}
$$

Note that the adjacency matrices of two isomorphic graphs are similar. Another naturally arising graph related matrix is the distance matrix of a connected graph $G, D_{G}$, defined 


$$
D_{G}(i, j)= \begin{cases}k, & d(i, j)=k \\ 0, & i=j\end{cases}
$$

Let $G[n]$ be a chemical graph on $n$ vertices with underlying molecule $L$. We explain our computational method in the following easy steps.

Step (i): Draw the chemical graph G in Hyperchem, where we obtain an hin file as an output.

Step (ii): Input the hin file to Topocluj by M. V. Diudea and his team and compute the adjacency and distance matrices of $G$.

Step (iii): Insert the adjacency and distance matrices of $G[n]$ in our programs developed in MATLAB. Our programs will compute certain distance-based topological indices such as the Szeged, revised Szeged, PI and eccentric-connectivity indices. More precisely, we obtain certain edge partitions for the Szeged, PI and revised Szeged indices for certain vertex partitions for eccentricity-based topological indices. The final computational values of these topological indices are obtained by using the aforementioned edge and vertex partitions. Our programs in MATLAB are accessible from the authors upon request.

\section{Distance-based topological indices}

4.1. Szeged, PI and revised Szeged indices. This section is devoted to the applications of our proposed technique developed in Section 3 to certain distance-based topological indices such as the Szeged index, the PI index and the revised Szeged index. The computation of exact analytical expressions for these topological indices for different classes of chemical graphs like fullerenes, carbon nanotubes etc. has been investigated in the literature. Mottaghi et al. [32] and Mehranian et al. [31] studied these distance-based topological indices for fullerenes. Ashrafi et al. [2] studied the PI and edge version of the Szeged index for certain infinite family of fullerenes. The extremal properties of the PI index with respect to different families of graph are studied in [40] and [39]. In Section 2, other related known results on these indices from both mathematical and chemical point of view are reported.

Now we turn our attention to further compute the Szeged, PI and revised Szeged indices for different families of polyominoes introduced in Section 2. In Step (iii) from Section 4, our proposed technique gives certain edge partitions of a chemical graph under consideration. For this class of topological indices, the corresponding edge partition is based on the triplet $\mathcal{N}(e)$ for any $e=u v \in E(G)$, where $G$ is the underlying graph. Our primary goal is to obtain these edge partitions for the polyomino chains $\mathbf{z}_{n}, \mathbf{c}_{n}, \mathbf{k}_{n}$ and $\mathbf{r}_{n}$. By using the MATLAB program we have developed we find the edge partition for these classes of chemical graphs. Tables 1, 2, 3 and 4 exhibit these edge partitions for $\mathbf{z}_{n}, \mathbf{c}_{n}, \mathbf{k}_{n}$ and $\mathbf{r}_{n}$, respectively. A graphical description of these types of vertices in different chains is depicted in Figure 4. We do not consider the polyomino chain $l_{n}$ as it has already been studied in terms of its alternative form i.e. $P_{n} \square P_{2}$, where $P_{n}$ is the path of length $n$ and $\square$ is the Cartesian product operation of graphs.

The 2-parametric polyomino chain system $P_{n}^{k}$ introduced in Section 1 is the generalization of all the polyomino chains we have introduced. The following lemma shows an important structural information about $P_{n}^{k}$ which helps us to show that the revised Szeged index and Szeged index coincide for the polyomino chains $\mathbf{z}_{n}, \mathbf{c}_{n}, \mathbf{k}_{n}$ and $\mathbf{r}_{n}$.

4.1. Lemma. Let $P_{n}^{k}$ be the 2-parametric polyomino chain system with $n, k \geq 1$. Then $P_{n}^{k}$ is a bipartite graph. 
Proof. Let $G$ be the graphs of 2-parametric polyomino chain system. It is known that a graph is bipartite graph if and only if it has no cycle of odd length. It is clear that there is no cycle of odd length in $G$. Thus $G$ is bipartite.

\begin{tabular}{|c|c|c|}
\hline Type & $(\nu(e \mid u), \nu(e \mid v), \nu(e \mid 0))$ where $u v \in E\left(\mathbf{z}_{n}\right)$ & No. \\
\hline 1 & $(4 n, 2,0)$ & 4 \\
\hline 2 & $(2 n+1,2 n+1,0)$ & 3 \\
\hline 3 & $(4 n-2 i+1,2 i+1,0), n \geq 1+i, \quad i=1,2,3, \ldots$ & 6 \\
\hline
\end{tabular}

Table 1. The values of $\mathcal{N}(e)$ for $e \in E\left(\mathbf{z}_{n}\right)$ and $n \geq 2$.

\begin{tabular}{|c|c|c|}
\hline Type & $(\nu(e \mid u), \nu(e \mid v), \nu(e \mid 0))$ where $u v \in E\left(\mathbf{c}_{n}\right)$ & No. \\
\hline 2 & $(6 n, 2,0)$ & 4 \\
\hline 2 & $(6 n-1,3,0)$ & 6 \\
\hline 3 & $(6 n-6 i+9,6 i-7,0), n \geq i, i=2,3, \ldots$ & 3 \\
\hline 4 & $(6 n-6 i+8,6 i-6,0), n \geq i, i=2,3, \ldots$ & 6 \\
\hline
\end{tabular}

Table 2. The values of $\mathcal{N}(e)$ for $e \in E\left(\mathbf{c}_{n}\right)$.

\begin{tabular}{|c|c|c|}
\hline Type & $(\nu(e \mid u), \nu(e \mid v), \nu(e \mid 0))$ where $u v \in E\left(\mathbf{k}_{n}\right)$ & No. \\
\hline 1 & $(8 n, 2,0)$ & 4 \\
\hline 2 & $(8 n-1,3,0)$ & 3 \\
\hline 3 & $(4,8 n-2,0)$ & 6 \\
\hline 4 & $(8 i-6,8 n-8 i+8,0), \quad n \geq i, \quad i=2,3,4, \ldots$ & 2 \\
\hline 5 & $(8 i-5,8 n-8 i+7,0), \quad n \geq i, \quad i=2,3,4, \ldots$ & 8 \\
\hline 6 & $(8 i-4,8 n-8 i+6,0), \quad n \geq i, \quad i=2,3,4, \ldots$ & 2 \\
\hline
\end{tabular}

Table 3. The values of $\mathcal{N}(e)$ for $e \in E\left(\mathbf{k}_{n}\right)$.

\begin{tabular}{|c|c|c|}
\hline Type & $(\nu(e \mid u), \nu(e \mid v), \nu(e \mid 0))$ where $u v \in E\left(\mathbf{r}_{n}\right)$ & No. \\
\hline 1 & $(10 n-4 i+4,4 i-2,0), i=1,2$ & 4 \\
\hline 2 & $(10 n-2 i+1,2 i+1,0), i=1,2$ & $2 i+1$ \\
\hline 3 & $(10 i-1,10 n-10 i+3,0), n \geq i+1, i=1,2,3, \ldots$ & 3 \\
\hline 4 & $(10 i-4,10 n-10 i+6,0), n \geq i+1, i=2,3, \ldots$ & 2 \\
\hline 5 & $(10 i+2,10 n-10 i, 0), n \geq i+1, i=1,2,3, \ldots$ & 2 \\
\hline 6 & $(10 i+4,10 n-10 i-2,0), n \geq i+1, i=1,2,3, \ldots$ & 8 \\
\hline
\end{tabular}

Table 4. The values of $\mathcal{N}(e)$ for $e \in E\left(\mathbf{r}_{n}\right)$. 

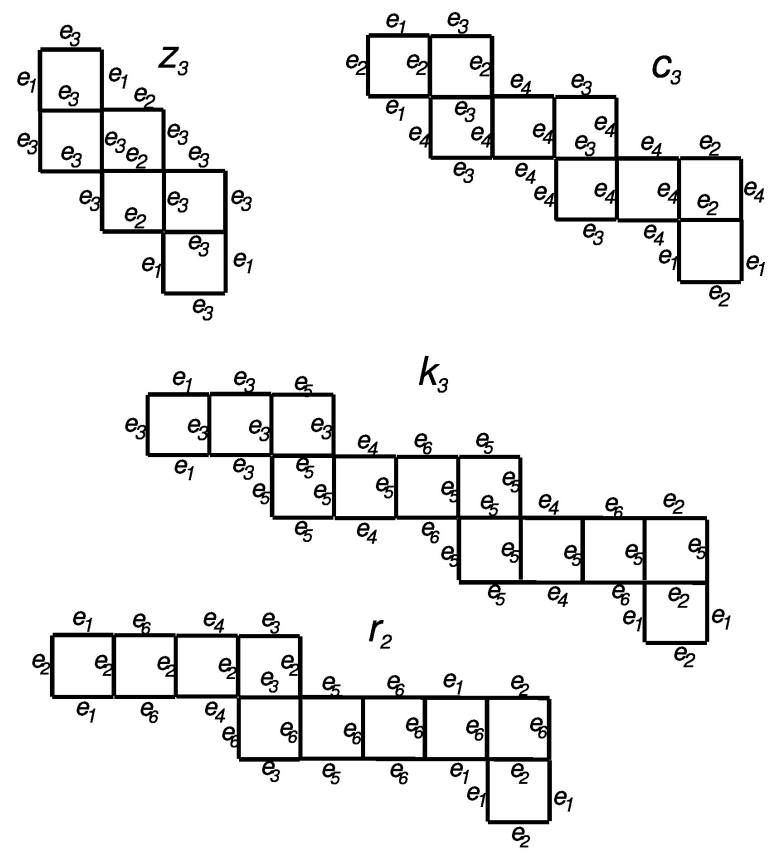

Figure 4. A description of different types of edges in polyomino chains.

The following well-known summations of polynomials functions are used to evaluate our exact analytical formulas:

$$
\sum_{i=m}^{n} 1=n+1-m, \quad \sum_{i=m}^{n} i=\frac{n(n+1)}{2}-\frac{m(m-1)}{2}=\frac{(n+1-m)(n+m)}{2} .
$$

4.2. Theorem. The Szeged index of different polyomino chains are computed as follows:

$$
\begin{aligned}
S z\left(\mathbf{z}_{n}\right) & =48 n^{3}-168 n^{2}+374 n-195, \\
S z\left(\mathbf{c}_{n}\right) & =54 n^{3}+54 n^{2}+39 n-9, \\
S z\left(\mathbf{k}_{n}\right) & =128 n^{3}+96 n^{2}+64 n-17, \\
S z\left(\mathbf{r}_{n}\right) & =250 n^{3}+150 n^{2}+99 n-27 .
\end{aligned}
$$

Proof. Let $G$ be the chemical graph of polyomino chains $\mathbf{z}_{n}$. Note that the number of edges in $G$ are $6 n+1$. Now we use the edge partition of $G$ in terms of $\mathcal{N}(e)$ for any edge $e=u v \in E(G)$. Table 1 shows this aforementioned edge partition of $G$. By definition of the Szeged index we have

$$
S z(G)=\sum_{e=u v \in E(G)} \nu(e \mid u) \nu(e \mid v) .
$$

By using edge partition of $G$ from Table 1, we obtain

$$
S z\left(\mathbf{z}_{n}\right)=4(6 n \times 2)+3((2 n+1) \times(2 n+1))+6 \sum_{\substack{i \in \mathbb{N} \\ n \geq 1+i}}((4 n-2 i+1) \times(2 i+1)) .
$$


By using the sequences of partial sums of certain series in above equation, we obtain

$$
S z\left(\mathbf{z}_{n}\right)=48 n^{3}-168 n^{2}+374 n-195 .
$$

Now assume $G$ to be the graph of the polyomino chain $\mathbf{c}_{n}$. The edge cardinality of $G$ is $9 n+1$. The edge partition for $G$, we need, is given in Table 2. By using this partition and the definition of Szeged index, we obtain

$$
\begin{aligned}
S z\left(\mathbf{c}_{n}\right)= & 4(4 n \times 2)+6((6 n-1) \times 3)+3 \sum_{\substack{1<i \in \mathbb{N} \\
n \geq i}}((6 n-6 i+9) \times(6 i-7))+ \\
& 6 \sum_{\substack{1<i \in \mathbb{N} \\
n \geq i}}((6 n-6 i+8) \times(6 i-6)) .
\end{aligned}
$$

A mechanical simplification of series in above equation yields,

$$
S z\left(\mathbf{c}_{n}\right)=54 n^{3}+54 n^{2}+39 n-9 .
$$

Next we assume $G$ to be the polyomino chain $\mathbf{k}_{n}$. The number of edges in this chain are $12 n+1$. By using the same argument as above and using the edge partition from Table 3 we obtain,

$$
\begin{aligned}
S z\left(\mathbf{k}_{n}\right)= & 4(8 n \times 2)+3((8 n-1) \times 3)+6(4 \times(8 n-2))+2 \sum_{\substack{1<i \in \mathbb{N} \\
n \geq i}}((8 i-6) \times \\
& (8 n-8 i+8))+8 \sum_{\substack{1<i \in \mathbb{N} \\
n \geq i}}((8 i-5) \times(8 n-8 i+7))+ \\
& 2 \sum_{\substack{1<i \in \mathbb{N} \\
n \geq i}}((8 i-4) \times(8 n-8 i+6)) .
\end{aligned}
$$

By using the sequences of partial sums of certain series in above equation, we obtain

$$
S z\left(\mathbf{k}_{n}\right)=128 n^{3}+96 n^{2}+64 n-17 .
$$

Finally, we assume $G$ to be the graph of polyomino chain $\mathbf{r}_{n}$. The edge set cardinality of $G$ is $15 n+1$. By definition of the Szeged index and Table 4, we obtain

$$
\begin{aligned}
S z\left(\mathbf{r}_{n}\right)= & 4 \sum_{i=1,2}((10 n-4 i+4) \times(4 i-2))+\sum_{i=1,2}(2 i+1)((10 n-4 i+4) \times \\
& (4 i-2))+3 \sum_{\substack{i \in \mathbb{N} \\
n \geq i+1}}((10 i-1) \times(10 n-10 i+3))+2 \sum_{\substack{i \in \mathbb{N} \\
n \geq i+1}}( \\
& (10 i-4) \times(10 n-10 i+6))+2 \sum_{\substack{i \in \mathbb{N} \\
n \geq i+1}}((10 i+2) \times(10 n-10 i))+ \\
& 8 \sum_{\substack{i \in \mathbb{N} \\
n \geq i+1}}((10 i+4) \times(10 n-10 i-2)) .
\end{aligned}
$$

By further simplifying the above equation, we obtain

$$
S z\left(\mathbf{r}_{n}\right)=250 n^{3}+150 n^{2}+99 n-27 .
$$

Which completes the proof.

4.3. Corollary. The revised Szeged index of a 2-parametric polyomino chain system is equal to its Szeged index. 
Proof. It is known that the Szeged and revised Szeged indices of a bipartite graph coincide. By Lemma 4.1, polyomino chain system is a bipartite graph which completes the proof.

4.4. Theorem. The PI index of different polyomino chains are computed as follows:

$$
\begin{aligned}
P I\left(\mathbf{z}_{n}\right) & =24 n^{2}+16 n+2, \\
P I\left(\mathbf{c}_{n}\right) & =54 n^{2}+24 n+2, \\
P I\left(\mathbf{k}_{n}\right) & =96 n^{2}+32 n+2, \\
P I\left(\mathbf{r}_{n}\right) & =150 n^{2}+40 n+2 .
\end{aligned}
$$

Proof. The proof is similar to the proof of Theorem 4.2. We, therefore, skip the proof of this result.

4.2. Wiener index. This short subsection focuses on computing Wiener index of polyomino chains. Graovac et al. [16] presented an algebraic way to compute the Wiener index of fullerene graph and computed it for fullerene graph on $10 n$ vertices. In what follows, the result by Graovac et al. for the Wiener index of $C_{10 n}$ fullerene graph is reported.

4.5. Theorem. [16] The Wiener index of $C_{10 n}$ fullerene graph is computed as follows:

$$
W\left(C_{10 n}\right)=\frac{100}{3} n^{3}+\frac{1175}{3} n-670 .
$$

In this subsection, we use our technique to generate the vertex partition of a chemical graph based on the transmission of any vertex and then compute its Wiener index.

\begin{tabular}{|c|c|c|}
\hline Type & $\operatorname{Tr}(v)$ where $v \in V\left(\mathbf{z}_{n}\right)$ & No. \\
\hline 1 & $4 n^{2}+4 n+1$ & 2 \\
\hline 2 & $4 n^{2}-(4 i-8) n+\left(2 i^{2}-6 i+11\right), n \geq i, \quad i=1,3,5, \ldots$ & 2 \\
\hline 3 & $4 n^{2}-(4 i-4) n+\left(2 i^{2}-2 i+3\right), n \geq i, i=2,4,6, \ldots$ & 2 \\
\hline 4 & $4 n^{2}-(4 i-4) n+\left(2 i^{2}-2 i+3\right), n \geq i, \quad i=1,3,5, \ldots$ & 2 \\
\hline 5 & $4 n^{2}-(4 i) n+\left(2 i^{2}+2 i+7\right), n \geq i, i=2,4,6, \ldots$ & 2 \\
\hline
\end{tabular}

Table 5. The values of $\operatorname{Tr}(v)$ for $v \in V\left(\mathbf{z}_{n}\right)$.

Our technique from Section 3 gives us a vertex partition of any chemical graph based on transmission of any vertex. By using this vertex partition and the modified definition of the Wiener index of any graph, i.e. $W(G)=\frac{1}{2} \sum_{1 \leq i \leq n} \operatorname{Tr}\left(v_{i}\right)$, we find analytical exact formulas of the Wiener index of aforementioned polyomino chains. Since the proof structure is same for all the chains, we only provide a proof for polyomino chain $\mathbf{z}_{n}$. Table 5 presents the edge partition of $\mathbf{z}_{n}$ based on the transmission of any vertex.

In the following theorem, the Wiener index of polyomino chains is calculated. We only prove it for $\mathbf{z}_{n}$ as other chains share similar structure of proof. 
4.6. Theorem. The Wiener index of different certain polyomino chains are computed as follows:

$$
\begin{aligned}
W\left(\mathbf{z}_{n}\right) & =\frac{16}{3} n^{3}+8 n^{2}+\frac{38}{3} n-1, \\
W\left(\mathbf{c}_{n}\right) & =18 n^{3}+18 n^{2}+21 n-3, \\
W\left(\mathbf{k}_{n}\right) & =\frac{128}{3} n^{3}+32 n^{2}+\frac{94}{3} n-5, \\
W\left(\mathbf{r}_{n}\right) & =\frac{250}{3} n^{3}+50 n^{2}+\frac{131}{3} n-7 .
\end{aligned}
$$

Proof. Let $G$ be the graph of polyomino chain $\mathbf{z}_{n}$. The number of vertices in $G$ are $4 n+2$. We can write the Wiener index of any graph $G$ as follows:

$$
W(G)=\frac{1}{2} \sum_{1 \leq i \leq n} \operatorname{Tr}\left(v_{i}\right) .
$$

By using this equation and the vertex partition of $\mathbf{z}_{n}$ bases on transmission of any vertex from Table 5, we obtain

$$
\begin{aligned}
W\left(\mathbf{z}_{n}\right)= & \frac{1}{2}\left[2\left(4 n^{2}+4 n+1\right)+2 \sum_{\substack{i \in \mathbb{N}^{-} \\
n \geq i}}\left(4 n^{2}-(4 i-8) n+\left(2 i^{2}-6 i+11\right)\right)+\right. \\
& 2 \sum_{\substack{i \in \mathbb{N}^{+} \\
n \geq i}}\left(4 n^{2}-(4 i-4) n+\left(2 i^{2}-2 i+3\right)\right)+2 \sum_{\substack{i \in \mathbb{N}^{-} \\
n \geq i}}\left(4 n^{2}-(4 i-4) n+\right. \\
& \left.\left.\left(2 i^{2}-2 i+3\right)\right)+2 \sum_{\substack{i \in \mathbb{N}^{+} \\
n \geq i}}\left(4 n^{2}-(4 i) n+\left(2 i^{2}+2 i+7\right)\right)\right]
\end{aligned}
$$

where $\mathbb{N}^{+}$(resp. $\mathbb{N}^{-}$) is the set of even (resp. odd) numbers. By using the sequences of partial sums of certain series, we obtain

$$
W\left(\mathbf{z}_{n}\right)=\frac{16}{3} n^{3}+8 n^{2}+\frac{38}{3} n-1 .
$$

\section{Eccentricity-based topological indices}

In this section we apply our computational technique to find exact analytical expressions of a number of eccentricity-based topological indices of chemical graphs. More precisely, we compute two eccentricity-based indices called the eccentric-connectivity index and the total eccentricity index of certain polyomino chains. The correlation coefficients of the eccentric-connectivity index for various physico-chemical properties of chemical compounds range from $95 \%$ to $99 \%$ [36]. In most of the cases observed in [36], the performance of the eccentric-connectivity index is far better than the Wiener and distance related topological descriptors. Researchers from theoretical and physical chemistry and mathematical chemistry have investigated various chemically and mathematically interesting properties of the eccentric-connectivity and the total eccentricity indices. We refer the reader to [1,9] and references therein, for their applications in chemistry and nanotechnology. We further refer the reader to [44] for various mathematical properties of these eccentricity-based topological indices.

It is worthy to mention here that the previous techniques of similar nature for computing distance-based topological of chemical graphs are restricted to the Szeged, PI and 
revised Szeged indices. Our technique is more general in this sense that besides computing aforementioned distance-based indices, one can compute the Wiener index and eccentricity related indices of chemical graphs.

\begin{tabular}{|c|c|c|c|}
\hline Type & $\varepsilon(v)$ & $d_{v}$ & No. \\
\hline 1 & $2 n+1$ & 2 & 2 \\
\hline 2 & $2 n$ & 3 & 2 \\
\hline 3 & $n+i, \quad 1 \leq i \leq n-1$ & 4 & 2 \\
\hline 4 & $n+i, \quad 1 \leq i \leq n$ & 2 & 2 \\
\hline
\end{tabular}

Table 6. The vertex partition of $\mathbf{z}_{n}$ for $n \geq 2$, based on $\varepsilon(v)$ and $d_{v}$ of any vertex $v \in V\left(\mathbf{z}_{n}\right)$.

\begin{tabular}{|c|c|c|c|}
\hline Type & $\varepsilon(v)$ & $d_{v}$ & No. \\
\hline 1 & $4 n+1$ & 2 & 2 \\
\hline 2 & $4 n$ & 2 & 2 \\
\hline 3 & $n+i, \quad n+1 \leq i \leq 3 n$ & 3 & 2 \\
\hline 4 & $n+i, \quad n+1 \leq i \leq 3 n-1$ & 2 & 1 \\
\hline 5 & $n+i, \quad n+1 \leq i \leq 3 n-1$ & 4 & 1 \\
\hline
\end{tabular}

Table 7. The vertex partition of $\mathbf{k}_{n}$ for $n \geq 2$, based on $\varepsilon(v)$ and $d_{v}$ of any vertex $v \in V\left(\mathbf{k}_{n}\right)$.
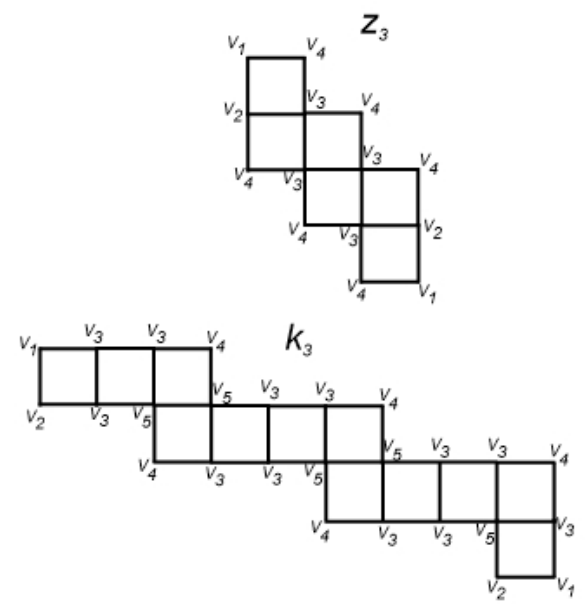

Figure 5. A description of vertex set partition with respect to eccentricities in polyomino chains $\mathbf{z}_{3}$ and $\mathbf{k}_{3}$.

In view of the definitions of the eccentric-connectivity and the total eccentricity indices, it is worth noticing that they are based on the vertex set partition based on eccentricity 
and degrees of any vertex of a graph. Our proposed technique in Section 3 yields us the related vertex partition of any graph. In our case, those partitions for different polyomino chains are presented in Tables $6,8,9,7,10$ and 11 . These partitions are useful to compute the eccentric-connectivity index and the total eccentricity index of certain polyomino chains. We notice that there are two different cases i.e. $n$ is even and $n$ is odd, for $\mathbf{c}_{n}$ and $\mathbf{r}_{n}$ whereas $\mathbf{z}_{n}$ and $\mathbf{k}_{n}$ can be managed in a single cases.

\begin{tabular}{|c|c|c|c|}
\hline Type & $\varepsilon(v)$ & $d_{v}$ & No. \\
\hline 1 & $3 n+1$ & 2 & 2 \\
\hline 2 & $3 n$ & 2 & 2 \\
\hline 3 & $3 n$ & 3 & 2 \\
\hline 4 & $\frac{3 n+2}{2}$ & 4 & 1 \\
\hline 5 & $\frac{3 n+2}{2}$ & 3 & 2 \\
\hline 6 & $\frac{3 n+2}{2}$ & 2 & 1 \\
\hline 7 & $\frac{3 n+4}{2}$ & 2 & 2 \\
\hline 8 & $\frac{3 n+4}{2}$ & 4 & 2 \\
\hline 9 & $n+i, \frac{n}{2}+3 \leq i \leq 2 n-1, i \neq 3 c\left(\frac{n}{2}+3\right)$ where $c \in \mathbb{N}$ & 4 & 1 \\
\hline 10 & $n+i, \frac{n}{2}+3 \leq i \leq 2 n-1, \quad i \neq 3 c\left(\frac{n}{2}+3\right)$ where $c \in \mathbb{N}$ & 3 & 2 \\
\hline 11 & $n+i, \frac{n}{2}+3 \leq i \leq 2 n-1, \quad i \neq 3 c\left(\frac{n}{2}+3\right)$ where $c \in \mathbb{N}$ & 2 & 1 \\
\hline 12 & $n+3 i\left(\frac{n+6}{2}\right), \quad 1 \leq i \leq \frac{4 n-4}{n+6}$ & 2 & 2 \\
\hline 13 & $n+3 i\left(\frac{n+6}{2}\right), \quad 1 \leq i \leq \frac{4 n-4}{n+6}$ & 4 & 2 \\
\hline
\end{tabular}

Table 8. The vertex partition of $\mathbf{c}_{n}$ for $n \mid 2$, based on $\varepsilon(v)$ and $d_{v}$ of any vertex $v \in V\left(\mathbf{c}_{n}\right)$.

\begin{tabular}{|c|c|c|c|}
\hline Type & $\varepsilon(v)$ & $d_{v}$ & No. \\
\hline 1 & $3 n+1$ & 2 & 2 \\
\hline 2 & $3 n$ & 2 & 2 \\
\hline 3 & $3 n$ & 3 & 2 \\
\hline 4 & $\frac{3 n+1}{2}$ & 2 & 1 \\
\hline 5 & $\frac{3 n+1}{2}$ & 4 & 1 \\
\hline 6 & $n+i, \frac{n+3}{2} \leq i \leq 2 n-1, \quad i \neq 3 c\left(\frac{n+3}{2}\right)$ where $c \in \mathbb{N}$ & 4 & 1 \\
\hline 7 & $n+i, \frac{n+3}{2} \leq i \leq 2 n-1, \quad i \neq 3 c\left(\frac{n+3}{2}\right)$ where $c \in \mathbb{N}$ & 3 & 2 \\
\hline 8 & $n+i, \frac{n+3}{2} \leq i \leq 2 n-1, \quad i \neq 3 c\left(\frac{n+3}{2}\right)$ where $c \in \mathbb{N}$ & 2 & 1 \\
\hline 9 & $n+3 i\left(\frac{n+3}{2}\right), \quad 1 \leq i \leq \frac{4 n-2}{n+3}$ & 2 & 2 \\
\hline 10 & $n+3 i\left(\frac{n+3}{2}\right), \quad 1 \leq i \leq \frac{4 n-2}{n+3}$ & 4 & 2 \\
\hline
\end{tabular}

Table 9. The vertex partition of $\mathbf{c}_{n}$ for $n \nmid 2$, based on $\varepsilon(v)$ and $d_{v}$ of any vertex $v \in V\left(\mathbf{c}_{n}\right)$.

Now we focus on computing the eccentric-connectivity and total eccentricity indices for aforementioned polyomino chains. We obtain the vertex partitions for these polyomino chains based on eccentricity and degree of a vertex. Tables 6 and 7 exhibited these vertex partitions for $\mathbf{z}_{n}$ and $\mathbf{k}_{n}$ respectively. In the similar spirit, Tables 8 and 9 show the required partitions for $\mathbf{c}_{n}$ for $n \mid 2$ and $n \nmid 2$ respectively and Tables 10 and 11 show the required partition for $\mathbf{c}_{n}$ for $n \mid 2$ and $n \nmid 2$ respectively. We use these partitions and the 


\begin{tabular}{|c|c|c|c|}
\hline Type & $\varepsilon(v)$ & $d_{v}$ & No. \\
\hline 1 & $5 n+1$ & 2 & 2 \\
\hline 2 & $5 n$ & 2 & 2 \\
\hline 3 & $5 n$ & 3 & 2 \\
\hline 4 & $\frac{3 n+6}{2}$ & 3 & 4 \\
\hline 5 & $n+i, \quad \frac{3 n}{2}+1 \leq i \leq \frac{3 n}{2}+2$ & 2 & 1 \\
\hline 6 & $n+i, \quad \frac{3 n}{2}+1 \leq i \leq \frac{3 n}{2}+2$ & 3 & 2 \\
\hline 7 & $n+i, \quad \frac{3 n}{2}+1 \leq i \leq \frac{3 n}{2}+2$ & 4 & 1 \\
\hline 8 & $n+i, \quad \frac{3 n}{2}+4 \leq i \leq 4 n-1, i \neq 5 c\left(\frac{3 n}{2}+4\right)$ where $c \in \mathbb{N}$ & 2 & 1 \\
\hline 9 & $n+i, \quad \frac{3 n}{2}+4 \leq i \leq 4 n-1, \quad i \neq 5 c\left(\frac{3 n}{2}+4\right)$ where $c \in \mathbb{N}$ & 3 & 2 \\
\hline 10 & $n+i, \quad \frac{3 n}{2}+4 \leq i \leq 4 n-1, \quad i \neq 5 c\left(\frac{3 n}{2}+4\right)$ where $c \in \mathbb{N}$ & 4 & 1 \\
\hline 11 & $n+5 i\left(\frac{3 n}{2}+4\right), \quad 1 \leq i \leq \frac{8 n-4}{3 n+8}$ & 3 & 4 \\
\hline
\end{tabular}

Table 10. The vertex partition of $\mathbf{r}_{n}$ for $n \mid 2$, based on $\varepsilon(v)$ and $d_{v}$ of any vertex $v \in V\left(\mathbf{r}_{n}\right)$.

\begin{tabular}{|c|c|c|c|}
\hline Type & $\varepsilon(v)$ & $d_{v}$ & No. \\
\hline 1 & $5 n+1$ & 2 & 2 \\
\hline 2 & $5 n$ & 2 & 2 \\
\hline 3 & $5 n$ & 3 & 2 \\
\hline 4 & $\frac{5 n+1}{2}$ & 3 & 2 \\
\hline 5 & $n+i, \quad \frac{3 n+1}{2} \leq i \leq 4 n-1, \quad i \neq 5 c\left(\frac{3 n+1}{2}\right)$ where $c \in \mathbb{N}$ & 2 & 1 \\
\hline 6 & $n+i, \quad \frac{3 n+1}{2} \leq i \leq 4 n-1, \quad i \neq 5 c\left(\frac{3 n+1}{2}\right)$ where $c \in \mathbb{N}$ & 3 & 2 \\
\hline 7 & $n+i, \quad \frac{3 n+1}{2} \leq i \leq 4 n-1, i \neq 5 c\left(\frac{3 n+1}{2}\right)$ where $c \in \mathbb{N}$ & 4 & 1 \\
\hline 8 & $n+5 i\left(\frac{3 n+1}{2}\right), \quad 1 \leq i \leq \frac{8 n-2}{3 n+1}$ & 3 & 4 \\
\hline
\end{tabular}

Table 11. The vertex partition of $\mathbf{r}_{n}$ for $n \nmid 2$, based on $\varepsilon(v)$ and $d_{v}$ of any vertex $v \in V\left(\mathbf{r}_{n}\right)$.

definitions of the eccentric-connectivity and total eccentricity indices to compute their exact analytical expressions for certain polyomino chains.

We record our calculations in the following theorems. We do not include the proofs of these results, as they are of similar nature as of results in the previous sections.

5.1. Theorem. The eccentric-connectivity index of different polyomino chains are computed as:

$$
\begin{aligned}
& \xi\left(\mathbf{z}_{n}\right)=48 n^{2}-20 n+4, \\
& \xi\left(\mathbf{k}_{n}\right)=72 n^{2}+20 n+16, \\
& \xi\left(\mathbf{c}_{n}\right)= \begin{cases}\frac{1}{2}\left(81 n^{2}+30 n+8\right), & n \mid 2 ; \\
\frac{1}{2}\left(81 n^{2}+30 n+5\right), & n \nmid 2,\end{cases} \\
& \xi\left(\mathbf{r}_{n}\right)= \begin{cases}\frac{1}{2}\left(225 n^{2}+26 n+8\right), & n \mid 2 ; \\
\frac{1}{2}\left(225 n^{2}+110 n+17\right), & n \nmid 2 .\end{cases}
\end{aligned}
$$


5.2. Theorem. The total eccentricity index of certain polyomino chains can be calculated by the following formulas.

$$
\begin{aligned}
\zeta\left(\mathbf{z}_{n}\right) & =6 n^{2}+6 n+2, \\
\zeta\left(\mathbf{k}_{n}\right) & =16 n^{2}+20 n+2, \\
\zeta\left(\mathbf{c}_{n}\right) & = \begin{cases}\frac{1}{2}\left(35 n^{2}+22 n+20\right), & n \mid 2 ; \\
\frac{1}{2}\left(27 n^{2}+18 n+3\right), & n \nmid 2,\end{cases} \\
\zeta\left(\mathbf{r}_{n}\right) & = \begin{cases}\frac{1}{2}\left(43 n^{2}+30 n+4\right), & n \mid 2 ; \\
\frac{1}{2}\left(75 n^{2}+30 n+3\right), & n \nmid 2 .\end{cases}
\end{aligned}
$$

\section{Degree-based topological indices}

This section is devoted to the study of certain degree-based topological indices of polyomino chains introduced in Section 2. We focus on computing the exact formulas of the $A B C$ index, the $G A$ index, the $A B C_{4}$ index and the $G A_{5}$ index of these polyomino chains. To study combinatorial and topological properties of certain graphs of specific interest, by computing their degree-based topological indices, is not new. In fact Hayat et al. [19] studied the degree-based topological indices of certain classes of silicate networks which have shown certain combinatorial interpretation of these class of networks. Imran et al. [21] considered certain interconnection networks and studied their degree-based topological indices. Moreover, degree-based indices of certain graphs of chemical interest are studied by Bača et al. [5] and Siddiqui et al. [37]. In the same spirit, we consider here various classes of polyominoes to calculate the analytically close formulas of four important degree-based topological indices of these classes of graphs.

To compute these topological indices of these infinite families of graphs, we derive two different partitions of the edge set of these graphs: one is based on degree of end vertices of any edge, and other is based on sum of degrees of neighbors of end vertices of each edge. Note that it is important to derive also the vertex and edge cardinalities of these polyomino chains. In Table 12, these cardinalities are presented.

\begin{tabular}{|c|c|c|}
\hline Polyomino chain & $\# V$ & $\# E$ \\
\hline $\mathbf{z}_{n}$ & $4 n+2$ & $6 n+1$ \\
\hline $\mathbf{c}_{n}$ & $6 n+2$ & $9 n+1$ \\
\hline $\mathbf{k}_{n}$ & $8 n+2$ & $12 n+1$ \\
\hline $\mathbf{r}_{n}$ & $10 n+2$ & $15 n+1$ \\
\hline
\end{tabular}

Table 12. The vertex and edge set cardinalities of different polyomino chains.

In the following tables, two important edge partitions are derived to compute the mentioned topological indices of polyomino chains. In Tables 13 and 14, these partition are derived for $n$-dimensional chain $\mathbf{z}_{n}$. The similar calculations for other polyomino chains are presented in the frequent tables. It is important to note that these edge partitions are used to prove the results derived on these topological indices. We provide the proofs of combinatorial meaning for our results.

Now we are in a good position to calculate these topological indices of different polyomino chains. In the following theorem, the $A B C$ index of certain polyomino chains are evaluated. 


\begin{tabular}{|c|c|c|c|c|c|}
\hline$\left(d_{u}, d_{v}\right)$ & $(2,2)$ & $(2,4)$ & $(2,3)$ & $(3,4)$ & $(4,4)$ \\
\hline No. & 2 & $4 n-4$ & 4 & 2 & $2 n-3$ \\
\hline
\end{tabular}

Table 13. The edge partition of $\mathbf{z}_{n}$ based on degrees of end vertices.

\begin{tabular}{|c|c|c|c|c|c|c|c|c|c|}
\hline$\left(S_{u}, S_{v}\right)$ & $(5,6)$ & $(6,11)$ & $(7,8)$ & $(8,5)$ & $(8,11)$ & $(8,12)$ & $(7,12)$ & $(11,12)$ & $(12,12)$ \\
\hline No. & 2 & 2 & 2 & 2 & 4 & $4 n-10$ & 2 & 2 & $2 n-5$ \\
\hline
\end{tabular}

Table 14. The edge partition of $\mathbf{z}_{n}$ based on sum of degrees of neighbors of end vertices.

6.1. Theorem. The ABC index of different polyomino chains can be calculated by the following formulas.

$$
\begin{aligned}
& A B C\left(\mathbf{z}_{n}\right)=\left(\frac{\sqrt{6}}{2}+2 \sqrt{2}\right) n-\frac{3 \sqrt{6}}{4}+\frac{\sqrt{15}}{3}+\sqrt{2}, \\
& A B C\left(\mathbf{c}_{n}\right)=\left(\frac{2 \sqrt{15}}{3}+\frac{\sqrt{6}}{4}+2 \sqrt{2}\right) n-\frac{\sqrt{15}}{3}-\frac{\sqrt{6}}{4}+2 \sqrt{2}, \\
& A B C\left(\mathbf{k}_{n}\right)=\left(\frac{2 \sqrt{15}}{3}+\frac{\sqrt{6}}{4}+2 \sqrt{2}+2\right) n-\frac{\sqrt{15}}{6}-\frac{\sqrt{2}}{2}+\frac{3 \sqrt{2}}{2}-\frac{\sqrt{6}}{4}-\frac{2}{3}+\sqrt{2}, \\
& A B C\left(\mathbf{r}_{n}\right)=\left(\frac{2 \sqrt{15}}{3}+\frac{\sqrt{6}}{4}+\frac{2}{3}+2 \sqrt{2}\right) n+\frac{3 \sqrt{2}}{2}+\frac{\sqrt{2}}{2}-\frac{\sqrt{15}}{6}-\frac{\sqrt{6}}{4}+\frac{2}{3}+\sqrt{2} .
\end{aligned}
$$

Proof. Let $G$ denote the polyomino chain $\mathbf{z}_{n}$. From Table 12, we know that the number of vertices and the number of edges in $\mathbf{z}_{n}$ are $4 n+2$ and $6 n+1$ respectively. By definition of the $A B C$ index, we have

$$
A B C(G)=\sum_{u v \in E(G)} \sqrt{\frac{d_{u}+d_{v}-2}{d_{u} \times d_{v}}} .
$$

Now we use the edge partition of $\mathbf{z}_{n}$ from Table 13 to further perform the necessary calculations. By using the definition of the $A B C$ index and the edge partition of $\mathbf{z}_{n}$ we obtain.

$$
\begin{aligned}
A B C\left(\mathbf{z}_{n}\right)= & 2 \sqrt{\frac{2+2-2}{2 \times 2}}+(4 n-4) \sqrt{\frac{2+4-2}{2 \times 4}}+4 \sqrt{\frac{2+3-2}{2 \times 3}}+ \\
& 2 \sqrt{\frac{3+4-2}{3 \times 4}}+(2 n-3) \sqrt{\frac{4+4-2}{4 \times 4}} .
\end{aligned}
$$

By simplifying the radical expressions, we obtain

$$
A B C\left(\mathbf{z}_{n}\right)=\left(\frac{\sqrt{6}}{2}+2 \sqrt{2}\right) n-\frac{3 \sqrt{6}}{4}+\frac{\sqrt{15}}{3}+\sqrt{2} .
$$

Note that the proofs for other polyomino chains are of similar structure, therefore, we skip them.

In the following result, without proof, we give exact analytical expressions for the $G A$ index of certain under-considered polyomino chains. 


\begin{tabular}{|c|c|c|c|c|c|}
\hline$\left(d_{u}, d_{v}\right)$ & $(2,2)$ & $(2,4)$ & $(2,3)$ & $(3,4)$ & $(4,4)$ \\
\hline No. & 2 & $2 n$ & $2 n+2$ & $4 n-2$ & $n-1$ \\
\hline
\end{tabular}

Table 15. The edge partition of $\mathbf{c}_{n}$ based on degrees of end vertices.

\begin{tabular}{|c|c|}
\hline$\left(S_{u}, S_{v}\right)$ & No. \\
\hline$(5,8)$ & 2 \\
\hline$(7,8)$ & 1 \\
\hline$(7,12)$ & $2 n-3$ \\
\hline$(10,12)$ & $4 n-6$ \\
\hline$(6,10)$ & 1 \\
\hline$(6,8)$ & 1 \\
\hline$(8,11)$ & 2 \\
\hline$(11,12)$ & 1 \\
\hline$(5,6)$ & 2 \\
\hline$(6,11)$ & 2 \\
\hline$(10,11)$ & 2 \\
\hline$(7,11)$ & 1 \\
\hline$(7,10)$ & $2 n-3$ \\
\hline$(12,12)$ & $n-1$ \\
\hline
\end{tabular}

Table 16. The edge partition of $\mathbf{c}_{n}$ based on sum of degrees of neighbors of end vertices.

6.2. Theorem. The GA index of different polyomino chains are calculated by the following formulas.

$$
\begin{aligned}
G A\left(\mathbf{z}_{n}\right) & =\left(\frac{8 \sqrt{2}}{3}+2\right) n-\frac{8 \sqrt{2}}{3}+\frac{8 \sqrt{6}}{5}+\frac{8 \sqrt{3}}{7}-1 \\
G A\left(\mathbf{c}_{n}\right) & =\left(\frac{16 \sqrt{3}}{7}+\frac{4 \sqrt{2}}{3}+\frac{4 \sqrt{6}}{5}+1\right) n-\frac{8 \sqrt{3}}{7}+\frac{4 \sqrt{6}}{5}+1 \\
G A\left(\mathbf{k}_{n}\right) & =\left(\frac{4 \sqrt{2}}{3}+\frac{4 \sqrt{6}}{5}+\frac{12 \sqrt{3}}{7}+4\right) n-\frac{2 \sqrt{2}}{3}-\frac{4 \sqrt{3}}{7}+\frac{6 \sqrt{6}}{5} \\
G A\left(\mathbf{r}_{n}\right) & =\left(\frac{4 \sqrt{2}}{3}+\frac{4 \sqrt{6}}{5}+\frac{16 \sqrt{3}}{7}+7\right) n-\frac{2 \sqrt{2}}{3}-\frac{4 \sqrt{3}}{7}+\frac{6 \sqrt{6}}{5}
\end{aligned}
$$

\begin{tabular}{|c|c|c|c|c|c|c|}
\hline$\left(d_{u}, d_{v}\right)$ & $(2,2)$ & $(2,4)$ & $(2,3)$ & $(3,3)$ & $(3,4)$ & $(4,4)$ \\
\hline No. & 2 & $2 n-1$ & $2 n+3$ & $3 n-1$ & $4 n-1$ & $n-1$ \\
\hline
\end{tabular}

Table 17. The edge partition of $\mathbf{k}_{n}$ based on degrees of end vertices.

Now we use the edge partitions based on the sum of degrees of end vertices of each edge. The following result presents the exact expressions for the $A B C_{4}$ index of certain polyomino chains. We do not give a proof of this result. 


\begin{tabular}{|c|c|}
\hline$\left(S_{u}, S_{v}\right)$ & No. \\
\hline$(5,5)$ & 1 \\
\hline$(5,6)$ & 1 \\
\hline$(5,8)$ & 2 \\
\hline$(5,9)$ & 1 \\
\hline$(6,8)$ & 1 \\
\hline$(6,9)$ & 1 \\
\hline$(6,11)$ & 1 \\
\hline$(7,9)$ & $2 n-2$ \\
\hline$(7,12)$ & $2 n-2$ \\
\hline$(8,9)$ & 2 \\
\hline$(8,11)$ & 1 \\
\hline$(9,10)$ & $2 n-3$ \\
\hline$(9,11)$ & 1 \\
\hline$(9,12)$ & $2 n-2$ \\
\hline$(10,10)$ & $n-1$ \\
\hline$(10,11)$ & 1 \\
\hline$(10,12)$ & $2 n-3$ \\
\hline$(12,12)$ & $n-1$ \\
\hline
\end{tabular}

Table 18. The edge partition of $\mathbf{k}_{n}$ based on sum of degrees of neighbors of end vertices.

6.3. Theorem. The $A B C_{4}$ index of different polyomino chains are computed as follows.

$$
\begin{aligned}
& A B C_{4}\left(\mathbf{z}_{n}\right)=\left(\frac{\sqrt{22}}{6}+2\right) n-\frac{5 \sqrt{3}}{2}+\frac{5 \sqrt{22}}{12}+\frac{\sqrt{30}}{15}+\frac{\sqrt{110}}{11}+\frac{\sqrt{374}}{11}+ \\
& \frac{\sqrt{357}}{21}+\frac{\sqrt{77}}{11}+\frac{\sqrt{182}}{14}+\frac{\sqrt{110}}{10}, \\
& A B C_{4}\left(\mathbf{c}_{n}\right)=\left(\frac{2 \sqrt{6}}{3}+\frac{\sqrt{357}}{21}+\frac{\sqrt{42}}{7}+\frac{\sqrt{22}}{12}\right) n+\frac{2 \sqrt{110}}{10}+\frac{\sqrt{182}}{28}-\frac{\sqrt{357}}{14}+ \\
& \frac{\sqrt{210}}{30}+\frac{\sqrt{374}}{22}+\frac{\sqrt{77}}{22}+\frac{\sqrt{30}}{5}+\frac{4 \sqrt{77}}{77}-\frac{3 \sqrt{42}}{14}-\frac{\sqrt{22}}{12}+ \\
& 2 \sqrt{\frac{19}{110}}+\frac{1}{2}-\sqrt{6} \\
& A B C_{4}\left(\mathbf{k}_{n}\right)=\left(\frac{4 \sqrt{323}}{19}+\frac{2 \sqrt{399}}{21}+\frac{3 \sqrt{10}}{10}+\frac{2 \sqrt{110}}{11}+\frac{\sqrt{14}}{2}+\frac{\sqrt{33}}{6}\right) n+ \\
& \frac{2 \sqrt{5}}{5}+\frac{3 \sqrt{11}}{11}+\frac{2 \sqrt{143}}{13}+\frac{2 \sqrt{42}}{7}+\frac{\sqrt{195}}{15}+\frac{\sqrt{255}}{17}-\frac{\sqrt{14}}{2}- \\
& \frac{4 \sqrt{323}}{19}+\frac{2 \sqrt{255}}{17}-\frac{\sqrt{399}}{21}-\frac{3 \sqrt{110}}{11}-\frac{\sqrt{33}}{6}, \\
& A B C_{4}\left(\mathbf{r}_{n}\right)=\left(\frac{2 \sqrt{2}}{3}+\frac{2 \sqrt{357}}{42}+\frac{4 \sqrt{170}}{30}+\frac{2 \sqrt{57}}{18}+\frac{2 \sqrt{6}}{6}+\frac{\sqrt{22}}{12}++\frac{8}{9}\right) n+ \\
& \frac{2 \sqrt{2}}{5}+\frac{\sqrt{30}}{10}+\frac{3 \sqrt{110}}{20}+\frac{\sqrt{78}}{18}+\frac{\sqrt{110}}{22}-\frac{2 \sqrt{2}}{3}-\frac{2 \sqrt{357}}{42}+ \\
& \frac{\sqrt{14}}{8}+\frac{\sqrt{30}}{12}+\frac{\sqrt{5}}{5}+\frac{\sqrt{374}}{44}-\frac{3 \sqrt{170}}{30}+\frac{\sqrt{22}}{11}-\frac{2 \sqrt{57}}{18}-\frac{2 \sqrt{6}}{6}+ \\
& \sqrt{\frac{19}{110}}-\frac{\sqrt{22}}{12}+\frac{1}{18}
\end{aligned}
$$




\begin{tabular}{|c|c|c|c|c|c|c|}
\hline$\left(d_{u}, d_{v}\right)$ & $(2,2)$ & $(2,4)$ & $(2,3)$ & $(3,3)$ & $(3,4)$ & $(4,4)$ \\
\hline No. & 2 & $2 n-1$ & $2 n+3$ & $6 n-1$ & $4 n-1$ & $n-1$ \\
\hline
\end{tabular}

Table 19. The edge partition of $\mathbf{r}_{n}$ based on degrees of end vertices.

In the similar spirit, we compute the exact analytical expressions of the $G A_{5}$ index for polyomino chains. We skip the proof due to structural similarity to the proofs of previous results.

6.4. Theorem. The $G A_{5}$ index of different polyomino chains are computed as follows.

$$
\begin{aligned}
& G A_{5}\left(\mathbf{z}_{n}\right)=\left(\frac{8 \sqrt{6}}{2}+2\right) n+\frac{4 \sqrt{30}}{11}+\frac{4 \sqrt{66}}{17}+\frac{8 \sqrt{14}}{15}+\frac{8 \sqrt{10}}{13}++\frac{16 \sqrt{22}}{19}+ \\
& \frac{8 \sqrt{21}}{19}+\frac{8 \sqrt{33}}{23}-4 \sqrt{6}-5 \\
& G A_{5}\left(\mathbf{c}_{n}\right)=\left(\frac{16 \sqrt{21}}{19}+\frac{8 \sqrt{30}}{11}+\frac{4 \sqrt{70}}{17}+1\right) n+\frac{8 \sqrt{10}}{13}+\frac{4 \sqrt{14}}{15}-\frac{12 \sqrt{21}}{19}- \\
& \frac{12 \sqrt{30}}{11}+\frac{\sqrt{15}}{4}+\frac{4 \sqrt{3}}{7}+\frac{8 \sqrt{22}}{19}+\frac{4 \sqrt{33}}{23}+\frac{4 \sqrt{30}}{11}+\frac{4 \sqrt{66}}{17}+ \\
& \frac{4 \sqrt{110}}{21}+\frac{\sqrt{77}}{9}-\frac{6 \sqrt{70}}{17}-1, \\
& G A_{5}\left(\mathbf{k}_{n}\right)=\left(\frac{3 \sqrt{7}}{4}+\frac{8 \sqrt{21}}{19}+\frac{12 \sqrt{10}}{19}+\frac{8 \sqrt{3}}{7}+\frac{4 \sqrt{30}}{11}+2\right) n++\frac{2 \sqrt{30}}{11}+ \\
& \frac{8 \sqrt{110}}{13}+\frac{3 \sqrt{5}}{7}+\frac{4 \sqrt{3}}{7}+\frac{2 \sqrt{6}}{5}+\frac{2 \sqrt{66}}{17}-\frac{3 \sqrt{7}}{4}-\frac{8 \sqrt{21}}{19}+ \\
& \frac{24 \sqrt{2}}{17}+\frac{4 \sqrt{22}}{19}-\frac{18 \sqrt{10}}{19}+\frac{3 \sqrt{11}}{10}-\frac{8 \sqrt{3}}{7}+\frac{2 \sqrt{110}}{21}-\frac{6 \sqrt{30}}{11}-1, \\
&\left(\frac{6 \sqrt{7}}{8}+\frac{8 \sqrt{21}}{19}+\frac{24 \sqrt{10}}{19}+\frac{8 \sqrt{3}}{7}+\frac{4 \sqrt{30}}{11}+3\right) n+\frac{2 \sqrt{30}}{11}+ \\
& \frac{12 \sqrt{10}}{13}+\frac{4 \sqrt{3}}{7}+\frac{2 \sqrt{6}}{5}+\frac{2 \sqrt{66}}{17}-\frac{6 \sqrt{7}}{8}-\frac{8 \sqrt{21}}{19}+\frac{12 \sqrt{2}}{17}+\frac{4 \sqrt{5}}{9}+ \\
& \frac{4 \sqrt{22}}{19}-\frac{18 \sqrt{10}}{19}+\frac{3 \sqrt{11}}{10}-\frac{8 \sqrt{3}}{7}+\frac{2 \sqrt{110}}{21}-\frac{4 \sqrt{30}}{11} . \\
& G A_{5}\left(\mathbf{r}_{n}\right)= \\
&=
\end{aligned}
$$




\begin{tabular}{|c|c|}
\hline$\left(S_{u}, S_{v}\right)$ & No. \\
\hline$(5,5)$ & 1 \\
\hline$(5,6)$ & 1 \\
\hline$(5,8)$ & 3 \\
\hline$(6,8)$ & 1 \\
\hline$(6,9)$ & 1 \\
\hline$(6,11)$ & 1 \\
\hline$(7,9)$ & $2 n-2$ \\
\hline$(7,12)$ & $2 n-2$ \\
\hline$(8,8)$ & 1 \\
\hline$(8,9)$ & 1 \\
\hline$(8,10)$ & 1 \\
\hline$(8,11)$ & 1 \\
\hline$(9,9)$ & $2 n-1$ \\
\hline$(9,10)$ & $4 n-3$ \\
\hline$(9,11)$ & 1 \\
\hline$(9,12)$ & $2 n-2$ \\
\hline$(10,11)$ & 1 \\
\hline$(10,12)$ & $2 n-2$ \\
\hline$(12,12)$ & $n-1$ \\
\hline
\end{tabular}

Table 20. The edge partition of $\mathbf{r}_{n}$ based on sum of degrees of neighbors of end vertices.

\section{Conclusion and limitation}

In this paper, we have developed a computational technique to compute certain topological indices of chemical graphs. We have applied our technique to compute those topological indices for certain infinite families of chemical graphs. Our results show that our technique is more diverse and possess a comparatively less algorithmic and combinatorial complexity.

Even though our technique is much general in nature, there are a number distancecum-adjacency indices which are not covered by it. These topological indices include the so-called the Gutman index and the Schultz index. It is still open to design a computational technique which also covers this class of topological indices. It might be a difficult problem for general graphs, but we believe it is possible to solve it for chemical graphs.

Acknowledgment We would like to thank the anonymous referees for their comments and suggestions which have considerably improved the quality and presentation of this paper.

\section{References}

[1] Ashrafi, A.R. Došlić, T. and Saheli, M. The eccentric connectivity index of $T U C_{4} C_{8}(R)$ nanotubes, MATCH Commun. Math. Comput. Chem. 65, 221-230, 2011.

[2] Ashrafi, A.R. Ghorbani, M. and Jalali, M. The PI and edge Szeged polynomials of an infinite family of fullerenes, Fullerenes, Nanotubes and Carbon Nanostructures 18 (3), 107-116, 2010.

[3] Aouchiche, M. and Hansen, P. On a conjecture about the Szeged index, European J. Combin. 31, 1662-1666, 2010.

[4] Arockiaraj M. Kavithah, S.R.J. and Balasubramanian, K. Vertex-cut methods for distancebased topological indices and its application to inorganic networks, J. Math. Chem. 54, 1728-1747, 2016. 
[5] Bača, M. Horváthová, J. Mokrišová, M. and Suhányiová, A. On topological indices of fullerenes, Appl. Math. Comput. 251, 154-161, 2015.

[6] Imran, M. and Hayat, S. On counting polynomials of certain polyomino chains, Bulg. Chem. Commun. 48, 332-337, 2016

[7] Diudea, M.V. Nanomolecules and nanostructures: polynomials and indices, University of Kragujevac, Kragujevac, 2010.

[8] Diudea, M.V. Ursu O. and Nagy, Cs.L. TOPOCLUJ, Babes-Bolyai University, Cluj, 2002.

[9] Dureja, H. and Madan, A.K. Superaugmented eccentric connectivity indices: new-generation highly discriminating topological descriptors for QSAR/QSPR modeling, Med. Chem. Res. 16, 331-341, 2007.

[10] Eliasi, M. and Taeri, B. Szeged index of armchair polyhex nanotubes, MATCH Commun. Math. Comput. Chem., 59 437-450, 2008.

[11] Estrada, E. Torres, L. Rodríguez, L. and Gutman, I. An atom-bond connectivity index: Modelling the enthalpy of formation of alkanes, Indian J. Chem., 37A, 849-855, 1998.

[12] The GAP Team, GAP, Groups, Algorithms and Programming, Lehrstuhl De fur Mathematik, RWTH, Aachen, 1992.

[13] Golomb, S.W. Polyominoes, Princeton University Press, Princeton, New Jersey, 1994.

[14] Graovac A. Ghorbani, M. and Hosseinzadeh, M.A. Computing fifth geometric-arithmetic index for nanostar dendrimers, J. Math. Nanosci. 1, 33-42, 2011.

[15] Graovac, A. and Hosseinzadeh, M.A. Computing ABC4 index of nanostar dendrimers, Optoelectron. Adv. Mater. Rapid Commun. 4, 1419-1422, 2010.

[16] Graovac, A. Ori, O. Faghani, M. and Ashrafi, A.R. Distance property of fullerenes, Iranian J. Math. Chem. 1, 5-15, 2010.

[17] Gutman, I. A formula for the Wiener number of trees and its extension to graphs containing cycles, Graph Theory Notes New York, 27, 9-15, 1994.

[18] Gutman, I. and Dobrynin, A.A. The Szeged index - a success story, Graph Theory Notes New York 34, 37-44, 1998.

[19] Hayat, S. and Imran, M. Computation of topological indices of certain networks, Appl. Math. Comput. 240, 213-228, 2014.

[20] HyperChem package Release 7.5 for Windows, Hypercube Inc., 1115 NW 4th Street, Gainesville, Florida 32601, USA, 2002.

[21] Imran, M. Hayat, S. and Malik, M.Y.H. On topological indices of certain interconnection networks, Appl. Math. Comput. 244, 936-951, 2014.

[22] Karelson, M. Molecular descriptors in $Q S A R / Q S P R$, Wiley, New York, 2000.

[23] Khadikar, P.V. Karmarkar, S. and Agrawal, V.K. A novel PI index and its applications to QSPR/QSAR studies, J. Chem. Inf. Comput. Sci., 41, 934-949, 2001.

[24] Klarner, D.A. Polyominoes, In: J. E. Goodman, J. O'Rourke, (eds.), Handbook of Discrete and Computational Geometry, CRC Press, Boca Raton, (1997), 225-242, Chapter 12.

[25] Liu, J.-B. and Cao, J. Applications of Laplacian spectra for n-prism networks, Neurocomputing, 198, 69-73, 2016.

[26] Liu, J.-B. Pan, X.-F. Yu, L. and Li, D. Complete characterization of bicyclic graphs with minimal Kirchhoff index, Discrete Appl. Math. 200, 95-107, 2016.

[27] Liu, J.-B. and Pan, X.-F. Minimizing Kirchhoff index among graphs with a given vertex bipartiteness, Appl. Math. Comput. 291, 84-88, 2016.

[28] Liu, J.-B. Wang, W.R. Zhang, Y.M. and Pan, X.-F. On degree resistance distance of cacti, Discrete Appl. Math. 203, 217-225, 2016.

[29] Liu, J.-B. Wang, C. Wang, S. and Wei, B. Zagreb indices and multiplicative Zagreb indices of Eulerian graphs, Bull. Malays. Math. Sci. Soc. DOI: 10.1007/s40840-017-0463-2.

[30] MATLAB and Statistics Toolbox Release 2012b, The MathWorks, Inc., Natick, Massachusetts, United States.

[31] Mehranian, Z. Mottaghi, A. and Ashrafi, A.R. The topological study of IPR fullerenes by studying their Szeged and revised Szeged indices, J. Theor. Comput. Chem. 11 (3), 547-559, 2012.

[32] Mottaghi, A. and Ashrafi, A.R. Topological edge properties of $C_{12 n+60}$ fullerenes, Beilstein J. Nanotechnol., 4, 400-405, 2013. 
[33] Pisanski, T. and Randić, M. Use of the Szeged index and the revised Szeged index for meauring network bipartivity, Discrete Appl. Math. 158, 1936-1944, 2010.

[34] Randić, M. On characterization of molecular branching, J. Amer. Chem. Soc. 97 (23), 6609-6615, 1975.

[35] Randić, M. On generalization of Wiener index for cyclic structures, Acta Chim. Slov. 49, 483-496, 2002.

[36] Sharma, V. Goswami, R. and Madan, A.K. Eccentric connectivity index: a novel highly discriminating topological descriptor for structure-property and structure-activity studies, J. Chem. Inf. Comput. Sci. 37, 273-282, 1997.

[37] Siddiqui, M.K. Imran M. and Ahmad A., On Zagreb indices, Zagreb polynomials of some nanostar dendrimers, Appl. Math. Comput. 280, 132-139, 2016.

[38] Vukičević, D. and Furtula, B. Topological index based on the ratios of geometrical and arithmetical means of end-vertex degrees of edges, J. Math. Chem. 46, 1369-1376, 2009.

[39] Wang, C. Wang, S. and Wei, B. Cacti with extremal PI index, Trans. Comb. 5, 1-8, 2016.

[40] Wang, S. and Wei, B. Multiplicative Zagreb indices of $k$-trees, Discrete Appl. Math. 180, $168-175,2015$.

[41] Wiener, H. Structural determination of the paraffin boiling points, J. Am. Chem. Soc. 69, $17-20,1947$.

[42] Xing, R. and Zhou, B. On the revised Szeged index, Discrete Appl. Math. 159, 69-78, 2011.

[43] Yarahmadia, Z. Ashrafi A.R. and Moradic, S. Extremal polyomino chains with respect to Zagreb indices, App. Math. Lett. 25, 166-171, 2012.

[44] Zhou, B. and Du, Z. On eccentric connectivity index, MATCH Commun. Math. Comput. Chem. 63, 181-198, 2010. 